\title{
Magnetic design for an electrodeless discharged lamp
}

\author{
T. Yanai, Y. Sakamoto, M. Honda, K. Takahashi, M. Nakano, \\ H. Kakehashi, and H. Fukunaga, Member, IEEE
}

\begin{abstract}
We proposed an analysis method for plasma in an electrodeless discharged lamp light-emitting by inductivelycoupled plasma, and a design of a power coupler was investigated by focusing on the electric power from the exciting coil to the plasma. We found that the power of the plasma was increased and of an Al stage was decreased with moving the core upward. This result suggests that the moving of the core upward is effective to reduce wasted power loss by eddy current and improves the lamp efficiency. In order to verify the result, we changed the position of the power coupler in the commercial lamp. Resultantly, the luminous flux of the lamp was increased by approximately $150 \mathrm{~lm}$ (lumen), when the power coupler was moved upward by $25 \mathrm{~mm}$ compared with the position of a commercial lamp. Therefore, we can conclude that our proposed method is applicable to the design of the lamp and suitable design is important to obtain high lamp efficiency.

Index Terms-Electrodeless discharged lamp, Inductivelycoupled plasma, High frequency excitation, electromagnetic fieldplasma interaction analysis.
\end{abstract}

\section{INTRODUCTION}

$\mathrm{R}$ ecently, an electrodeless discharged lamp, which has some industrial advantages such as long lifetime and relatively high lamp efficiency, is used for a light source with relatively high luminous flux such as a street lamp ${ }^{[1]}$. In particular, the electrodeless lamp with an inner coil ${ }^{[2,3]}$ shows high lamp efficiency because driven frequency of several hundred $\mathrm{kHz}$ is lower than $\mathrm{RF}$ band for outer coil type ${ }^{[2]}$. Although the lamp with an inner coil is one of hopeful candidates for new light source, its lamp efficiency is approximately $90 \mathrm{~lm} / \mathrm{W}$ and relatively low compared with those for conventional lamps such as high-pressure sodium ones $(110 \sim 130 \mathrm{~lm} / \mathrm{W})$ and metal halide ones $(70 \sim 130$ $\mathrm{lm} / \mathrm{W}$ ), which prevents it from for a further spread. In this type of lamp, as the electric power is transmitted to the plasma through the magnetic coupling, the state of the plasma is strongly affected by magnetic factors such as material, position and shape of magnetic core. A suitable magnetic design that could supply much electric power to the plasma is important to obtain the high lamp efficiency because the light emission of the lamp originates from the plasma. Therefore, development of an analysis method for Inductively-Coupled Plasma (ICP) in the lamp is effective to design the suitable lamp shape. Although some simulation

T. Yanai(corresponding author), Y. Sakamoto, M. Honda, K. Takahashi, M. Nakano, and H. Fukunaga are with the Division of Electrica Engineering and Computer Science, Graduate school of Engineering, Nagasaki University, Nagasaki, 852-8521, Japan (telephone: +81-95-8192554, e-mail: t-yanai@nagasaki-u.ac.jp).

K. Kakehashi is with the Panasonic Electric Works Information Systems Co., Ltd., 1048, Kadoma, Osaka, 571-8686, Japan method for ICP were reported ${ }^{[4-6]}$, analysis target of them is RF-driven ICP such as plasma process and ion engine. Therefore, we have developed a simulation method for low frequency driven ICP in the lamp using Finite Element method. In this study, in order to investigate the possibility of improvement in the lamp efficiency, we focused on the transmitted power to the plasma and investigated the suitable design of the core in the lamp.

\section{ANALYSIS PROCEDURE}

\section{A. Model lamp}

Figure 1(a) shows an analysis object, which is a spherical electrodeless discharge lamp (150W type) with an inner coil produced by Panasonic Electric Works, Ltd. The lamp was composed of a bulb and a power coupler. A phosphor material was applied inner surface of the bulb. Ar gas and amalgam (Hg-metal compound) were enclosed in the bulb, and gas pressures under lighting were 23.2 $\mathrm{Pa}$ and $0.93 \mathrm{~Pa}$ for $\mathrm{Ar}$ and $\mathrm{Hg}$, respectively. The power coupler was consisted of an $\mathrm{Al}$ die-cast, $\mathrm{Cu}$ tube, a ferrite core and exciting coil. The $\mathrm{Cu}$ tube and the $\mathrm{Al}$ die-cast were used for cooling of the core and a stage of the lamp, respectively.

\section{B. Analysis procedure}

Figures 1(b)-(d) and 2 show a model and the flow for the analysis, respectively. 3D-analysis was carried out for the bulb and the power coupler considering axial symmetry of the lamp (Fig.1 (b)). For magnetic field analysis, vacuum area and infinity boundary one were also modeled (Fig.1 (c)), and then we divided those area to small elements (Fig.1 (d)). An element size is approximately $1 \times 1 \mathrm{~mm}$ and total element number is approximately 30,000. After the settings of the input electric power $P_{\text {in }}$ of $150 \mathrm{~W}$ and the initial value of the plasma (electron) density $n_{e 0}$ of $10^{19} \mathrm{~m}^{-3}$, the high frequency exciting current $I_{0}(135 \mathrm{kHz})$ of $2 \mathrm{~A}$ was given to the exciting coil, and the FEM analyses of (1) the flux density $B$, (2) the electric field $E$, (3) the electron temperature $T_{e}$ and (4) the ionization frequency of the gas atom $v_{i}$ were carried out in this order. $T_{e}$ and $v_{i}$ were obtained by the experimental result between $E$ and $T_{e}$ in positive column and the following equation, respectively.

$$
v_{i}=2 \sqrt{\frac{2 e}{\pi m_{e}}} p a V_{i^{2}}^{\frac{3}{2}}\left(1+2 \frac{e V_{i}}{k T_{e}}\right) \exp \left(-\frac{k T_{e}}{e V_{i}}\right) \text {, }
$$

where $e, m_{e}, a, p, V_{i}$, and $k$ are the elementary electric charge $\left(=1.602 \times 10^{-19} \mathrm{C}\right)$, the electron mass $\left(=9.109 \times 10^{-31}\right.$ 
$\mathrm{kg})$, the

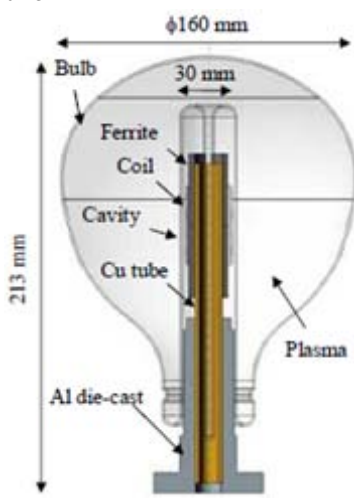

(a) Model lamp

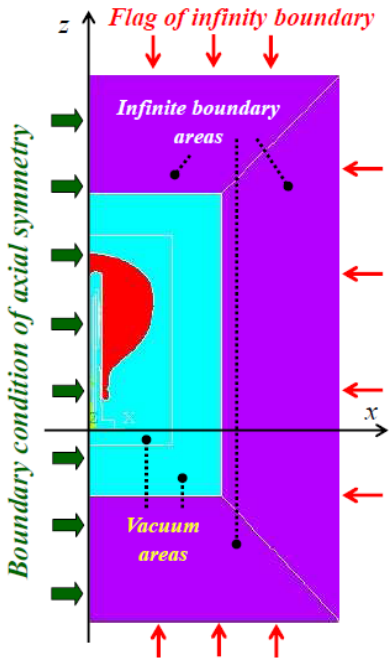

(c) Boundary conditions

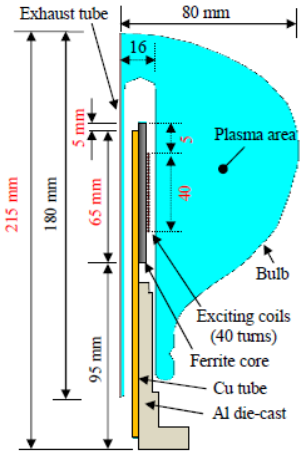

(b) Analysis model

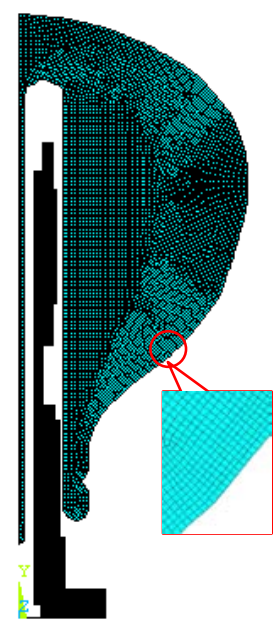

(d) Meshed model
Fig.1 Analysis objects and model. A spherical electrodeless discharge lamp (150W type) with inner coil produced by Panasonic Electric Works, Ltd. was used as based model in this study.

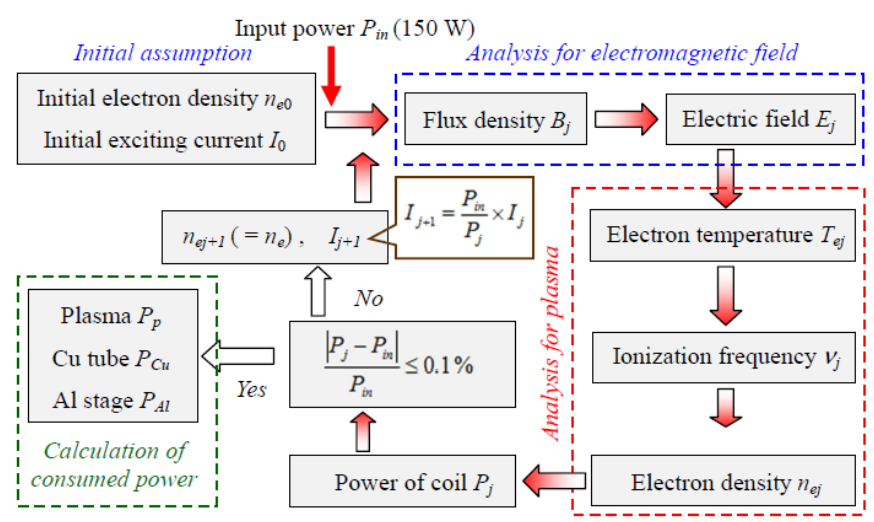

Fig.2 Analysis flow.

initial gradient of ionization for $\mathrm{Hg}(=110 / \mathrm{Pa} \cdot \mathrm{m})$, gas pressure of $\mathrm{Hg}(=0.93 \mathrm{~Pa})$, ionization energy of $\mathrm{Hg}(=10.42$ $\mathrm{eV})$, and the Boltzmann constant $\left(=1.381 \times 10^{-23} \mathrm{~J} / \mathrm{K}\right)$, respectively. Subsequently, (5) the analysis of $n_{e}$ was carried out by solving the following diffusion equation.

$$
\frac{D_{a}}{r} \frac{\partial}{\partial r}\left(r \frac{\partial n_{e}}{\partial r}\right)+D_{a} \frac{\partial^{2} n_{e}}{\partial z^{2}}+v_{i} n_{e}=0,
$$

where $D_{a}$ is bipolar diffusion coefficient. $D_{a}$ in weaklyionized plasma is given as

$$
D_{a} \approx \frac{k \cdot \mu_{i}}{e} T_{e}
$$

where $\mu_{i}$ is the mobility of $\mathrm{Hg}^{+}\left(=0.42 \mathrm{~m}^{2} / \mathrm{V} \cdot \mathrm{s}\right)$. As the obtained distribution of $n_{e}$ differs from the initial value $n_{e 0}$, the exciting current $I$ was changed and the series of analyses from (1) to (5) were repeated until the power of the coil $P_{\text {coil }}$ converges to $P_{i n}$. After conversion of $P_{\text {coil }}$, the powers related to an eddy current loss in the plasma, the $\mathrm{Cu}$ tube and the Al stage areas were calculated and defined as $P_{P}, P_{C u}$ and $P_{\text {Al }}$, respectively.

In this analysis, an effect of the powers on the position of the core and the power coupler was investigated.

\section{RESULTS AND DISCUSSIONS}

\section{A. Analyzed Results}

Dependences of the $P_{p}, P_{C u}$ and $P_{A l}$ on the top position of the core were shown in the Fig.3. Figure 4 shows schematic representation of analysis model. The position of $0 \mathrm{~mm}$ corresponds to the position for the commercial lamp. Increase in $P_{p}$ and decrease in $P_{A l}$ were observed by moving the core upward. As distance between the bottom of the core and the top of the Al stage is increased when the position is moved to upward, an eddy current in the Al stage is decreased. Figure 5 shows eddy current per unit volume in the power coupler. It is found that large eddy current flow at the top of the $\mathrm{Al}$ stage and $\mathrm{Cu}$ tube around the bottom of the core. Therefore, a design of the power coupler for reduction in the eddy current in the $\mathrm{Cu}$ tube and the $\mathrm{Al}$ stage enables us to improve the lamp efficiency, and we found that increase in the distance between the bottom of the core and the top of the $\mathrm{Al}$ stage is one of effective design to obtain the high lamp efficiency.

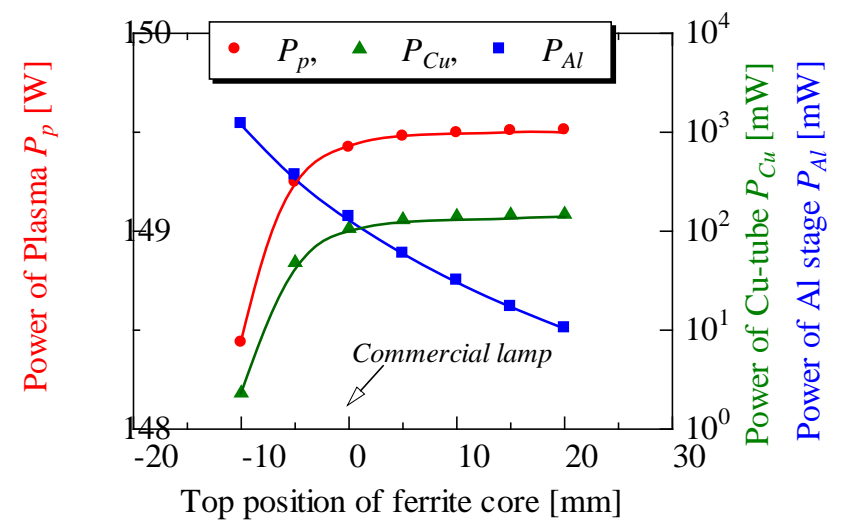

Fig. 3 Dependences of transmitted power to plasma $P_{p}$, consumed powers in Cu-tube $P_{C u}$ and Al stage $P_{A l}$ on the top position of the ferrite core. The position for a commercial lamp is $165 \mathrm{~mm}$. 


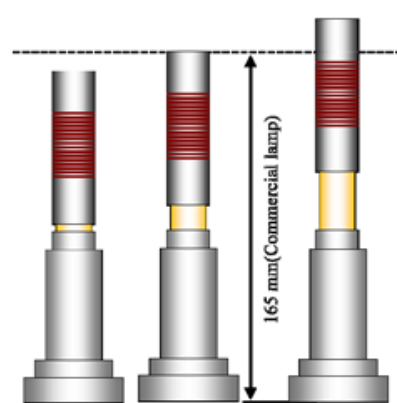

Fig.4 Schematic representation of change in the top position of the ferrite core.

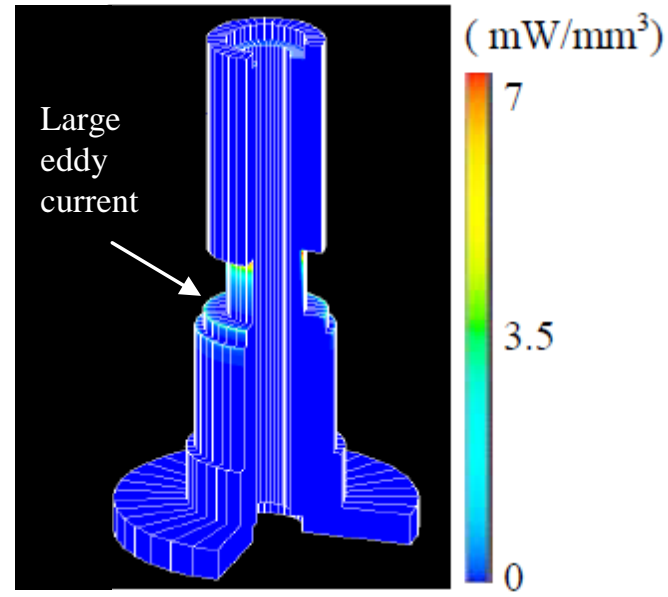

Fig. 5 Eddy current per unit volume in the power coupler.

\section{B. Experimental Result}

In order to verify the analyzed result that upward-moved core is effective to improve the lamp efficiency, the position of the power coupler in the commercial lamp was changed from $-5 \mathrm{~mm}$ to $25 \mathrm{~mm}$, and the luminous flux $L F$ was measured at each position. As it is difficult to change the length of $\mathrm{Cu}$ tube in the commercial lamp, we changed top position of the power coupler. Figure 6 shows dependence of $L F$ on the top position of the core, together with the analyzed result for the power of the plasma $P_{p}$. $L F$ was increased with moving the power coupler upward and a good correlation was observed between $P_{p}$ and $L F$.

From abovementioned results, we can conclude that our proposed method is one of hopeful analysis method for the lamp and the design of increasing $P_{p}$ is effective to improve the lamp efficiency.

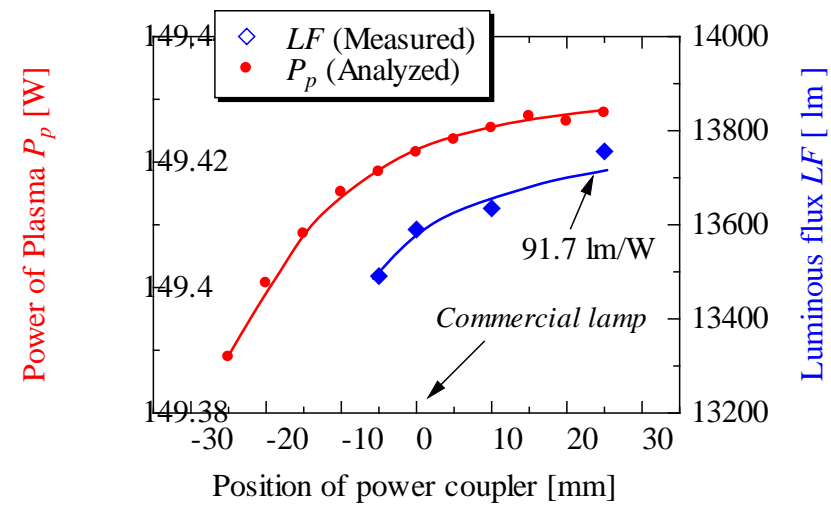

Fig. 6 Dependences of $P_{p}$ and measured luminous flux $L F$ on the position of the power coupler.

\section{CONCLUSION}

In this paper, we developed a simulation method for low frequency driven ICP in the spherical electrodeless discharged lamp with an inner coil and investigated the suitable lamp design to improve in the lamp efficiency. The obtained results are summarized as follows;

(1) Increase in the distance between the bottom of the core and the top of the $\mathrm{Al}$ stage is one of effective design to obtain the high lamp efficiency because large eddy current flow at the top of the $\mathrm{Al}$ stage.

(2) Large eddy current in $\mathrm{Cu}$ tube was observed around the bottom of the core, and we need to reduce the eddy current in this part especially to improve in the lamp efficiency.

(3) Luminous flux of the commercial lamp was increased with moving the power coupler upward and we confirmed a good correlation was observed between analyzed power of plasma and measured luminous flux of the lamp.

Our future work is detail investigation of the design in the bulb and the power coupler of the lamp to obtain high lamp efficacy.

\section{REFERENCES}

[1] T. Yasuda, M. Bessho, S. Naoki, A, Saito, A. Maehara, A. Okada, H. Asano, and J. Hasegawa, "Light sources and lighting circuits," J. Light \& Vis. Env., vol. 34, pp. 57-59, 2010.

[2] J. Bethenod, and A. Claude, US Patent \#2,030,957, 1936.

[3] T. Arakawa, K. Seki, K. Katase, K, Hashimotodani, and A. Hochi, "Light sources and lighting circuits," J. Light \& Vis. Env., vol. 30, pp. 34-38, 2006.

[4] S. K. Nam, and D. J. Economou, "Two-dimensional simulation of a miniaturized inductively coupled plasma reactor”, J. Appl. Phys., vol. 95, pp. 2272-2277, 2004.

[5] S. Zhao, X. Xu, X. Li, and Y. Wang, "Fluid simulation of the E-H mode transition in inductively coupled plasma”, J. Appl. Phys., vol. 105, pp. 083306-083316, 2009.

[6] T. Takahashi, Y. Takao, K. Eriguchi, K. Ono, "Numerical and experimental study of microwave-excited microplasma and micronozzle flow for a microplasma thruster”, Phys. Plasmas, vol. 16, pp. 083505-083519, 2009. 